\title{
Dynamic Simulation in Virtual Environments as an Evaluation Tool for Architectural Design
}

\author{
Şule Taşlik and Bülent Özgüç*
}

Prediction and evaluation of future performance of buildings are essential aspects of an efficient design process. Thispaper discusses dymamic simulation as a prediction and evaluation tool for architectural design. It is proposed that since buildings are living entities, whole life cycles of buildings sbould be dynamically simulated in a bigbly visualized virtual environment to evaluate the future performance of prospective designs. The media of arcbitectural design (paper-based media: drawings and physical scale models; and digital media) are compared in terms of their capacity to support dynamic simulations. Some promising application areas are mentioned and some proposals are presented for the future use of dynamic simulations in virtual environments.

\section{Introduction}

Although buildings have static structures, everything else related to architecture is dynamic. Environmental factors like sun, wind, and humiditychange with time. People move through the buildings and they interact with them in numerous ways. Use pattems are likely to change in time and in some probabilities several events like fires, earthquakes, or floods may happen.

Evaluation of architectural designs against the criteria such as environmental factors, human factors, economy, etc. is an essential part of an efficient design process. Since architectural systems are rathercomplex to comprehendand to make predictions about future performance, a means of predicting the performance of buildings is needed. Dynamic simulation is building a model, that incorporates time, and using this model to test or experiment with designs. To conduct a dynamic simulation for architectural design, a medium is needed to "virtually" build and live in a building before the actual construction. A virtual environment or world in this sense is

1. The contents of some medium; 2. A space that exists in the mind of its creator, often manifested in some medium; 3. A description of a collection of objects in a space, and the rules and relationships governing those objects [1].

Although the idea of simulation is not new for architectural design,

\footnotetext{
* Bilkent University, Faculty of Art, Design and Architecture, Department of Interior Architecture and Environmental Design, 06533, Bilkent, Ankara, Turkey. 'Corresponding author
}

simulation media (should) change according to the developments in technology. In this paper, first we explain why simulation is a suitable methodology for architectural design. Then, we discuss modeling media in terms of dynamic simulation and finally we present some proposals for the future use of dynamic simulation in virtual environments (VE) as an evaluation tool for architectural design.

\section{Simulation as a Methodology for Architectural Design}

Computer simulation is a branch of applied mathematics that is widely used by many disciplines. It is used in different senses to study a variety of systems that may be classified as continuous vs. discrete, deterministic vs. stochastic or dynamic vs. steady state. Simulation is used within many areas, so it is considered to be a methodology [2]. We claim that simulation is a methodology that well suits the needs of evaluation of architectural designs. Architectural design should be considered as an interface between people and buildings to respond to the needs of people. Unfortunately, it is observed that this fundamental role of design as an interface has been forgotten for some of the cases. It is not only because of the ignorance of the architects, but also of the complexity of the factors that are essential to design but difficult to incorporate the design process. These factors are becoming more and more complex in time; therefore computers should be used to simulate them [3].

Simulation is a method that is well suited to the needs of the building design. Because:

1. Unlike in other industries, it is impossible to build a first realistic 
prototype in building design, because nomally every building is unique.

2. Real experiments can be dangerous (e.g. fire evacuation, themal comfort, etc.)

3. Since buildings are living entities, forecasting models are needed to analyze periods in building life cycle in a compressed format.

4. Mathematical modeling of most of the architectural systems does not lend themselves to practical anaiytical or numeric solutions.

\section{Modeling Media in Architectural Design}

In architectural design, modeling is a process, either mental or extemalized, of translating conceptual ideas into visual forms. Atthough at its root the idea of modeling has been the same throughout the history, it has taken on many forms of expression. These expressions are mainly the result of technological advances in producing imagery. In this section, we compare paper-based design media (drawings and physical models) with digital design media in terms of their capacity to support dynamic simulations.

Paper-based drawings and physical scale models are old and widely used types of modeling media in architectural design. However, they are inefficient to support simulations for architectural design. Drawings and physical scale models are rather abstract representations that are not related to the context of use. Therefore, they are not proper for testing and refinement of designs. Jones, quoted in Mitchell's book, claims that by paper-based media, designers focus on visual articulation and tend to ignore other things that they fail to represent [3].

Computer graphics and CAAD revolutionized modeling media in architectural design, since a digital model of a design is capable of representing a design much better than paper-based media. The main difference between digital media and paper-based media is in the concept of simulation. Digital media enables testing buildings before they are built. Nevertheless, not all types of digital design media are suitable for dynamic simulation. Following Mitchell and McCullough's categorization of digital design media according to media dimension: one (words, texts, and sounds), two (images, drafted lines, maps, etc.), three (wireframe, surface, and solid models), and multi dimensional media (motion models and animation) [4], it is clear that only the multi dimensional media has the capability for the representation of the fourth dimension in architecture, that is time.

Among the multi dimensional media, virtual reality (VR) represents the latest development in the process of digitalization of architectural design, which initially started with CAAD. CAAD models grow into virtual environments in the following order.

Static perspective renderings, from wireframe models to textured surface renderings

$\downarrow$

Animated noninteractive walk-throughs

$\downarrow$

Interactive screen-based virtual environments

$\downarrow$

Immersive virtual environments

Virtual environments offer new ways for the simulation and visualization of architectural concepts by the help of the rapid progress in computer technology. Computer graphics pioneers predict that in near future, the display and computational capability to produce images that are both physically accurate and perceptually indistinguishable from real worid scenes will be available. This means that at that time simulation technology will reach such a level of capability that there will be no difference with real and virtual wortds and verification tools might be needed to avoid confusion between them [5] [6].

How can architects benefit from this technology? Although there is no limit for the application areas of dynamic simulations in virtual environments for architectural design, at the current state of simulation technology two areas seem especially promising for near future: evaluation of user-building interaction and visualization of environmental factors.

Evaluation of user-building interaction in virtual environments can be achieved through the use of virtual humans. Virtual humans are computer-generated, graphically displayed entities that represent real humans. Several major industries such as aerospace, automotive, and shipbuilding industries have already embraced them. Virtual humans have been used for cockpit design, aircraft crew cabin layout and instrumentarion evaluation, automobile seat and passenger comfort studies, and space station construction planning. They are used to test the fit, reach, and motions of people in vehicles and environments. They also permit assessment of movement parterns of individuals and groups of people [7].

The interest in and the development of virtual humans have increased in the last decade [8]. This can be due to two major factors. First, the advances in computer graphics technology have provided the performance and speed necessary to efficienty duplicate and visualize human motion. Second, design professionals are becoming increasingly aware that the human factor is a critical design element that must be accounted for in every phase of the product life cycle [7].

Badler claims that in future digital copies of specific individuals (not only their shapes but their mannerisms and behaviors) will be available. Virtual humans will not just look like realistic people; they will have their personalities, reactions, and emotions [9]. Such models can be utilized in architectural design to evaluate dynamic and experiential user needs. By combining the virtual model of his/her design with virtual humans, an architect can understand the possible results of interaction between the proposed building and its prospective users. At a very simple level, accessibility and safety considerations can be analyzed according to the different user types (female, male, elderly, children, disabled, etc.) Autonomous virtual humans can be used in analyzing events like evacuation, panic, or wayfinding in buildings. At the most advanced level, using specially designed software architects can be immersed in their designs "becoming" the actual user. For example, an architect designing a house for an elder person with glaucoma (an eye disability for elderly) will really benefit from seeing the virtual model of the building "through the eyes" of the client.

The other possible application area of dynamic simulation in virtual environments is the visualization of environmental factors. Computers can currently relatively easily simulate environmental performance of buildings like thermal behavior, structural behavior, acoustics, and lighting. Nevertheless, experiencing design behavior is yet only possible with VR. Conventional simulation tools leave architects with a large amount of data in a difficult to understand format. On the other hand, VR interfaces can display data in a way much closer to their nature than by means of other symbols such as words and numbers. Design behavior can be represented as colors, sounds, motion-models and so on and these can be directly mapped on the virtual model. Interaction in a three-dimen- 
sional space, navigation, and instant feedback are the other benefits of visualization in VEs.

Computational Fluid Dynamics (CFD) and auralization of sound (i.e. rendering spatialized sound based on acoustic modeling) are two main types of application of visualization in VEs for architectural design. CFD can be used to simulate air movements, heat transfer, or the progress of a potential fire in buildings. In the process of sound auralization in VEs reverberation paths from a sound source to a listener are computed and the results are visualized in a virtual environment. Such simulations can be used by architects to solve acoustical problems in design.

\section{The Use of Dynamically Simulated Virtual Models in Architectural Design: Some Problems and Proposals}

Although the benefits of CAAD tools and techniques are well known in architecture, dynamic simulations in virtual environments are not much used for architectural applications. This is probably due to the relative recentness of the subject. However, increased complexity in architectural design processes and clients' ever-growing demands toward results force architects to explore and adapt new technologies. Design criteria should be taken into account, preferably in the early stage of design and not during analysis later in the process. Otherwise, there is always a danger that designs will be more and more based on intuition and aesthetic considerations rather than a realistic understanding of future performance of proposed buildings.

We suggest that in order to meet these demands a virtual model of a proposed building, that dynamically simulates all the phases of a building life span from the brief to the demolition, should be produced in an early stage of design development. Such a virtual model can be used for several types of applications from design to marketingand facilities management.

Object-oriented technology can be utilized in the implementation of virtual building models. The basic idea of object-oriented programming is to combine the data describing the object and the operations related to it. Object-oriented programming well suits to the needs of architects, because architects always have to deal with objects (components of buildings, environmental factors, users, etc.) and the relations between the objects (which also can be defined as objects). Object-oriented programming enables definition of objects in hierarchies with inheritance capability. This is a reasonable way to produce a virtual building model as a system capable of providing intelligent and dynamic response. In fact, object-oriented programming dates back to first simulation programming languages like Smalltalk and Simula in 1960s and it has been increasingly used since early 1990 s by CAAD software vendors. However, functionality of current object-oriented CAAD sofware is very limited compared to that of virtual prototypes used in engineering design.

Virtual prototypes can be definedas dynamic, interactive, often immersive three-dimensional CAD models utilizing VR technology and developed to analyze product designs. Such computer-based models are usually referred as to be "intelligent" because theyrespond to the situations beyond mere display. They capture product content, generate and simulate manufacturing processes, and predict product behavior. When once built, a virtual prototype can be used to support diverse activities such as costestimation, marketing, and material-requirements planning throughout the product'slife cycle. Although the idea of using VRin industry is not a new one, VR technology has only recently matured enough to enable engineering design applications. Several companies and government agencies are currently investigating the application of VR techniques to their design and manufacturing processes. Virtual prototypes of product designs are replacing the real ones [10].

As the cost of VR systems drops, other applications that once seemed cost-prohibitive are becoming a possibility, for example, simulators that allow service providers to experience the impact of their work on their customers. By studying the results of potential customers' interaction with a virtual prototype designers can understand the advantages and disadvantages of a proposed design. Another advantage of virtual prototypes is explaining concepts and ideas to non-technical persons. Designers usually have difficulty in this process. Three-dimensionality and interactivity of virtual prototypes help designers in explaining design ideas to the others [11].

The benefits of virtual prototypes in engineering design have already been proved and we can leam much from them for the implementation of virtual building models that will also act as unique prototypes for proposed buildings.

\section{Comparison of Dynamically Simulated Virtual Models with Conventional CAAD}

In this section, we compare conventional-drafting based $C A A D$ and $V R$. Of course, both of them covers a lot of different systems and with the rapid pace of development in software industry, the terms tend to blur easily. However, our distinction between the two types of models depends mainly on the latter's capability of providing dynamic and intelligent response. Then, the possible advantages of a three-dimensional, interactive, dynamically simulated virtual model for architectural design compared to conventional CAAD are discussed below.

Simulation of building performance for the whole life span of a proposed building saves the client and architect time and money and enhances the quality of design. Buildings are expensive entities to build and maintain and the deficiencies in a building design may cost much through the whole building life. Even a small increase in the operating and maintenance costs of buildings per square meter will be totaled much through the years. Many of these deficiencies can be eliminated by dynamic time simulations in virual environments.

In architectural design, most of the important decisions (orientation, circulation, functional layout, etc.) are made at the early stages of design. Nevertheless, most of the CAAD software does not support these stages of design development. CAAD software is traditionally used for producing construction documents after the preliminary design is complete. The digital model produced at this stage is rarely used after the construction of the building. On the other hand, functionality of dynamically simulated virtual models has already been proved in the early phases of engineering design [11]

One may claim that such applications for architecture will cost much, but the rapid decreases in the costs of computer products suggest that they can be feasible even for the smallest offices. Screen-based VR can be used for most of the applications instead of the immersive type and PCs that are widely used in architectural offices may suffice for most of the applications.

Moreover, in conventional drafting-based $C A A D$, modeling, analysis and visualization processes follow a linear sequence. Analyses are applied after the modeling is complete and visualization occurs at the latest stages of design only for presentation purposes. However, a virtual model can be more flexible compared to physical models and other CAAD models. 
Simulation, evaluation, and modification will be able to take place within seconds through a highly interactive user interface with real time feedback functionality. Therefore, modeling, analysis and visualization will occur concurrently. The benefits of this property are clear: the shortening of the design cycle and quality improvements due to gained information (Figure 1).

\section{Proposals}

Although their uses are very promising, production and utilization of dynamic simulations in VEs for architectural design require further study tion of evaluation tools with design tools has often been very difficult because of the difference of the intemal information representations. VEs will not be an exception to these problems. Since a great deal of data is needed to perform simulations, VEs should allow gathering data from different nodes. In order to read data from different systems, the VR system and the other digital tools of the architect have to be compatible. Market conditions have been already forcing the vendors of CAAD software to produce compatible products [12], but we suggest that these efforts should also embrace VR systems.

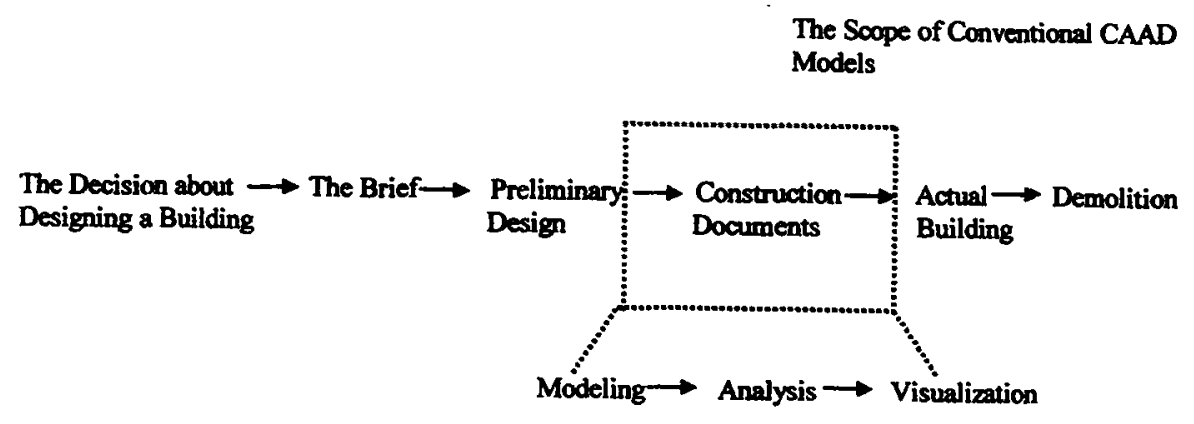

The Scope of Dynamically Simulated

Virtual Models

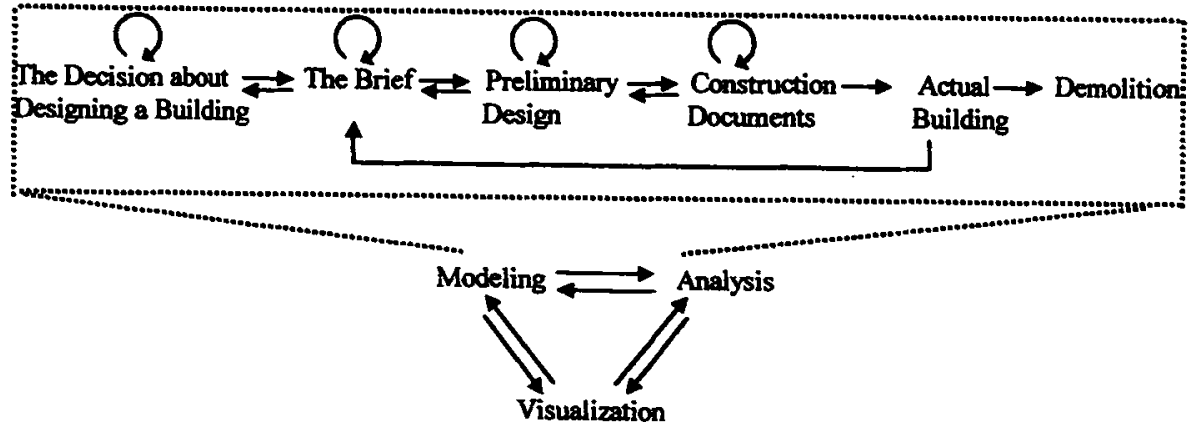

Figure 1. Comparison of Dynamically Simulated Virtual Models with Conventional CAAD

on different fronts. First of all, we suggest that architects should redesign their design processes according to the new possibilities. They should redefine their expectations from CAAD and its way of use in their professional activities. Computers should be viewed as tools to expand and explore understanding of proposed building and its relation to the environment and the users rather than drafting machines. The other important considerations for the efficient use of dynamic simulations in VEs for architectural design are discussed below.

\section{Compatibility and Standards}

Compatibility and standardization have always been problems for the users of CAAD software. Architects usually have to use two or more software packages to produce a high-level output such as a complete animation. Designs are produced with a two-dimensional drafting software, and then an animation or rendering software is used to complete the task. Lack of compatibility between different computer aided design software renders the use of them together impossible. Moreover, integra-
Since VR technology is relatively new, it can be easier to solve these problems at this stage of development. Development of standards for the VEs on the web is an important step toward compatibility and standardization of VEs. Virtual Reality Modeling Language (VRML) is the result of such efforts. VRML 97 became an Intemational Organization for Standardization (ISO) standard in 1997 and now forms the basis for many new works [13].

\section{Interface Design}

The computer is a tool that can provide several kinds of services, but to benefit from these services users must communicate with the computer system. Therefore, interface design is an imporant factor for obtaining maximum benefit from the CAAD software. We discuss that atthough CAAD systems relieve architects of repetitive drafting tasks, some of the conventional CAAD interfaces are difficult to use and waste time and energy. For example, we may compare the mental effort needed to draw a line with a pen on a paper and the mental effort needed to draw a similar 
line with such a $C A A D$ system. The CAAD system requires much attention to activate the "line" command and to specify several parameters. Attention shifts from the design task to interaction task that consumes time and energy. We suggest that a design system should be quick and intuitive, capturing the flow of the concepts as quickly and naturally as possible. Advantages of VR interfaces were mentioned before in this paper. In general, it can be said that a virtual model is more adaptive to design processes of architects than any digital model. We believe that VR interfaces can eliminate most of the problems in conventional CAAD interfaces. Nevertheless, to obtain maximum benefits from dynamic simulations in VEs for architectural design, specific needs of this task should also be addressed. For example, what should be the properties of an ideal interface to obtain simulation data from different nodes and to integrate them into the geometric model? Or, how the visualization of simulation results should interface to architects? Since simulation data and resulting visualizations should be shared by the different participants of building process, how a common ground can be provided for different applications? Answers to these and similar questions lie in the interface design research and these considerations deserve more attention for further work.

\section{The Need for Collection and Distribution of Information}

Any type of computer simulation necessitates information to be gathered. When this information is complex and/or divergent, as in our case, this process can be time-consuming and expensive. Moreover, this data needs to be continuously updated. Interdisciplinary work is needed for most of the instances and we cannot expect that single architectural offices, no matter how large they are, will be willful or capable of conducting these processes. We suggest that the information required for the production of dynamic simulations should be drawn from Environmental Design Research (EDR). EDR has been producing a huge amount of data already that is waiting to be used. Researchers of environmental design often complain that their works do not have impact on the architectural community. However, this is not only due to the ignorance of the architects, but it is also due to the inconvenient format of the outputs of EDR for architects. In fact, some architects still do not know what EDR means and they do not have time to learn much in the rapid market conditions. Simulation in virtual environments can effectively utilize research data and present the results in a way familiar to the architects. Architects, as designers working in three-dimensions, will really benefit from the three-dimensional and interactive visualization in VEs.

We also suggest that government agencies, research institutions and professional organizations should support and fund research and development on this topic. Potential applications of dynamic simulations in VEs are so rapidly evolving that perhaps in near future buildings will be checked against regulations in dynamically simulated VEs.

Simulation in VEs will also help to increase the methodological knowledge in architectural design. Simulation enlarges the insight in the overall system and effects of a certain alteration on one or more variables can be observed easily. Therefore, verification of some design theories can be possible in VEs.

Simulation models and programs can be distributed over the Internet easily. In this way, even the architects having the simplest computer configurations can conduct simulations by connecting to the high performance computers preserving simulation model in a distant place. This not only saves the time, but it also lets the architect manipulate the simulation model to suit his/her specific problems or needs. The simulation data can be marketed over the network, for example, in the form of virual human profiles derived from behavioral research. In order to preserve the copyrights, several measures can be taken such as presentation of low-resolution data at the trial stages.

\section{Validation of Simulation Models and Representations for}

\section{Visualization}

Validation of simulation model is a common step for every type of computer simulation. This process is required to ensure that the mathematical model successfully represents the reality. Discussing dynamic simulations in VEs for architectural design, a more philosophical question lies in the "True Model" concept. Visual experience and perception comes into the scene for this case and certain difficulties in modeling time, colors and textures in relation to the scale factor of physical dimensions should be adressed. Since, it is yet impossible to simulate the real visual experience, it is offered to "catch a likeness" that reveals a key aspect of a prospective design, rather than trying to simulate the whole visual experience [14].

For the effective use of visualization in architectural design some points should be considered. First of all, visualization techniques and representations should be well adapted to the needs of architects. Visualization research originated from the scientific community's efforts to cope with the huge amount of scientific data. Therefore, specific representations have already produced in scientific visualization for the technically skilled audience. Since architects are not scientists, ease of use is a key factor for the efficient architectural visualizations. Visualization designed for architects should enable them to get the information they need on their specific problems, make sense of it, and reach decisions easily in a relatively short time. Interfaces should allow easy manipulation of data and representations should not lead to misinterpretations.

The lack of standards and ways to integrate visualization across multiple applications [15] renders these tasks difficult. Last but not least, we suggest that since the media of visualization are relatively new, potential benefits of using them need to be well understood. Architects should not use this medium as a replica of paper, but should explore the new possibilities offered.

\section{References}

1. A. ROWELl: A Definition of Virtual Reality. Computer Grapbics World. Vol. 20.2, 1997, pp. 21.

2. R. E. NANCE and C. M. OVERSTREET: Computer Simulation: Achieving Credible Experimental Results in Virtual Environments. Simulation Digest. Vol. 24.3, 1995, pp. 40-50.

3. T. C. MITCHELL: Redefining Designing from Form to Experience. New York: Van Nostrand Reinhold, 1993.

4. W. J. MITCHELL and M. MCCULLOUGH: Digital Design Media. New York: Van Nostrand Reinhold, 1991.

5. D. GREENBERG: Outlook on Computer Graphics. Computer. Vol. 31.1, 1998, pp. 36.

6. R. JAIN: Real Reality. IEEE Computer Graphics and Applications. JanFeb. 2000 , pp. 40-41.

7.J. S. MILLER: Digital Humans in the Simulated Product Life Cycle. IIE Solutions. Vol. 30.3, 1998, pp. 24-30.

8. B. ROBERTSON: Virtual Humans at Work. Computer Graphics World. Vol. 20.7, 1997, pp. 33-39. 
9. N. I. BADLER: Animation 2000++. IEEE Computer Grapbics and Applications. Jan-Feb. 2000, pp. 40-41.

10. M. HALPERN: Driving Toward Feature-based Virtual Prototyping. Computer Graphics World. Vol. 20.9, 1997, pp. 23-25.

11. M. HARDING: Virtual Reality the Art of Engineering. Design Engineering. April 1998, pp. 19-21.

12. B.J. NOVTISKI: AEC Systems 2000 Takes Architects for a Ride. Arcbitectural Record. V. 189.6, 2000, pp. 205-206.
13. R. NADEAU: Building Virtual Worlds with VRML. IEEE Computer Grapbics and Applications. Vol. 19.2, 1999, pp. 18-29.

14. B. SENYAPIII: The True Model Concept in Computer Generated Simulations Used in Architectural Design. Full Scale Modeling in the Age of Virtual Reality. Proc. of the $6^{\text {th }}$ European Full Scale Modeling Association Conference. Vienna: Iris-Isis Pub, 1996. 133-40.

15. N. GERSHON and S. G. EICK: Information Visualization. IEEE Computer Graphics and Applications. Vol. 17.4, 1997, pp. 29-31. 